\title{
Simulation For 2D Flows And Water Quality in Curvilinear Coordinates
}

\author{
Wei Wenli G.W.Rong Y.L. Liu \\ Institute of Water Conservancy and Hydraulic Engineering, \\ Xi'an University of Technology \\ Xi'an, China, 710048 \\ weiwenli@xaut.edu.cn
}

\begin{abstract}
This paper presents a numerical method to simulate the 2D tidal flow and water quality under the orthogonal curvilinear coordinates. In order to overcome the computational difficulties in natural rivers, such as the complicated boundary figures, the great disparity between length and width of computational domain, etc., orthogonal boundary-fitted grid is used, the irregular domain in physical plane is transformed into a rectangular domain in transformed plane, and the depth averaged momentum equations and mass equation are rewritten and discretized based on the alternating direction implicit finite difference scheme in curvilinear coordinates. Practical application of the method is illustrated by an example for the Yangtze River in the vicinity of Nanjing city. A fair agreement between the values measured and computed demonstrates the validity of the method developed.
\end{abstract}

Keywords : orthogonal curvilinear coordinate; numerical simulation; flow; water quality; tidal river

\section{INTRODUCTION}

The discharge of polluted water is becoming an increasing threat to the resources in tidal areas. The numerical simulation of 2D tidal flow and water quality is the basis of water resource planning and management. In recent years, although considerable attention has been focused on this problem, the computational difficulties in natural tidal rivers remain to be a big problem to modelers, such as the complicated boundary figures, the great disparity between length and width, etc.

In the following, a 2D numerical model based on the alternating direction implicit finite difference scheme for tidal flow and water quality under the curvilinear coordinate is suggested, the irregular domain in physical plane is transformed into a rectangular domain in computational plane, and the depth-averaged momentum equations and mass equation are rewritten and discretized in curvilinear coordinates. A practical application of the model is illustrated by an example for the Yangtze River in the vicinity of Nanjing city.

\section{MATHEMATICAL MODEL}

\section{A. Generation of Orthogonal Curvilinear Grids}

Generating a grid in an arbitrary physical domain involves a coordinate transformation from the physical plane $(\mathrm{x}, \mathrm{y})$ to the computational plane $(\xi, \eta)$. This is done here by solving a system of Poisson equation ${ }^{[1]}$.

$$
\begin{aligned}
& a x_{\xi \xi}-2 \beta x_{\xi \eta}+\not x_{\eta \eta}=p_{1} \\
& a y_{\xi \xi}-2 \beta y_{\xi \eta}+y_{\eta \eta}=p_{2}
\end{aligned}
$$

where

$$
a=x_{\eta}^{2}+y_{\eta}^{2}, \quad \beta=x_{\xi} x_{\eta}+y_{\xi} y_{\eta}, \quad \gamma=x_{\xi}^{2}+y_{\xi}^{2}
$$

and

$$
p_{1}=-J^{2}\left(x_{\xi} p+x_{\eta} Q\right), \quad p_{2}=-J^{2}\left(y_{\xi} p+y_{\eta} Q\right) \text {. }
$$

In the above equations, $\mathrm{J}$ is the Jacobian $\left(=x_{\xi} y_{\eta}-x_{\eta} y_{\xi}\right)$ of transformation; and $\mathrm{P}$ and $\mathrm{Q}$ are control functions which can be chosen to provide a denser distribution of points in certain regions.

In Eq. (1), determining the function of $P$ and $Q$ is often difficult problem. Wei Wen- $\mathrm{Li}^{[2,3]}$ has proposed a new method to determine them more efficiently. By using the method, a desired boundary-fitted curvilinear coordinate grid can be automatically generated.

\section{B. Transformed governing equation}

Since the numerical computations are performed on a orthogonal mesh, it is necessary to convert Cartesian formulas into transformed equations written in terms of the boundaryfitted orthogonal curvilinear coordinates $\xi$ and $\eta$. The transformed shallow water governing equations are ${ }^{[3,4]}$

$$
\frac{\partial z}{\partial t}+\frac{1}{g_{\xi} g_{\eta}} \frac{\partial}{\partial \xi}\left(H U g_{\eta}\right)+\frac{1}{g_{\xi} g_{\eta}} \frac{\partial}{\partial \eta}\left(H V g_{\xi}\right)=0
$$

\footnotetext{
* Corresponding author: weiwenli@xaut.edu.cn
} 


$$
\begin{aligned}
& \frac{\partial U}{\partial t}+\frac{U}{g_{\xi}} \frac{\partial U}{\partial \xi}+\frac{V}{g_{\eta}} \frac{\partial U}{\partial \eta}+\frac{U V}{g_{\xi} g_{\eta}} \frac{\partial g_{\xi}}{\partial \eta}- \\
& \frac{V^{2}}{g_{\xi} g_{\eta}} \frac{\partial g_{\eta}}{\partial \xi}+g \frac{U \sqrt{U^{2}+V^{2}}}{C^{2} H}-f V+\frac{g}{g_{\xi}} \frac{\partial z}{\partial \xi} \\
& =\varepsilon\left(\frac{1}{g_{\xi}} \frac{\partial A}{\partial \xi}-\frac{1}{g_{\eta}} \frac{\partial B}{\partial \eta}\right)
\end{aligned}
$$

$\frac{\partial V}{\partial t}+\frac{U}{g_{\xi}} \frac{\partial V}{\partial \xi}+\frac{V}{g_{\eta}} \frac{\partial V}{\partial \eta}+\frac{U V}{g_{\xi} g_{\eta}} \frac{\partial g_{\eta}}{\partial \xi}-$

$\frac{U^{2}}{g_{\xi} g_{\eta}} \frac{\partial g_{\xi}}{\partial \eta}+g \frac{V \sqrt{U^{2}+V^{2}}}{C^{2} H}+f U+\frac{g}{g_{\eta}} \frac{\partial z}{\partial \eta}$

$=\varepsilon\left(\frac{1}{g_{\eta}} \frac{\partial A}{\partial \eta}+\frac{1}{g_{\xi}} \frac{\partial B}{\partial \xi}\right)$

$$
\begin{aligned}
& \frac{\partial(H \varphi)}{\partial t}+\frac{1}{g_{\xi} g_{\eta}} \frac{\partial}{\partial \xi}\left(H U g_{\eta} \varphi\right)+\frac{1}{g_{\xi} g_{\eta}} \frac{\partial}{\partial \eta}\left(H V g_{\xi} \varphi\right) \\
& =\frac{1}{g_{\xi} g_{\eta}} \frac{\partial}{\partial \xi}\left(D_{\xi} H \frac{g_{\eta}}{g_{\xi}} \frac{\partial \varphi}{\partial \xi}\right)+\frac{1}{g_{\xi} g_{\eta}} \frac{\partial}{\partial \eta}\left(D_{\eta} H \frac{g_{\xi}}{g_{\eta}} \frac{\partial \varphi}{\partial \eta}\right)+S_{\varphi}
\end{aligned}
$$

where

$$
\begin{gathered}
A=\left[\frac{\partial}{\partial \xi}\left(U g_{\eta}\right)+\frac{\partial}{\partial \eta}\left(V g_{\xi}\right)\right] / g_{\xi} g_{\eta} \\
B=\left[\frac{\partial}{\partial \xi}\left(V g_{\eta}\right)+\frac{\partial}{\partial \eta}\left(U g_{\xi}\right)\right] / g_{\xi} g_{\eta} \\
g_{\xi}=\sqrt{x_{\xi}^{2}+y_{\xi}^{2}} \\
g_{n}=\sqrt{x_{\eta}^{2}+y_{\eta}^{2}} .
\end{gathered}
$$

$\mathrm{t}$ is time; $\mathrm{z}$ is water surface elevation; $\mathrm{H}$ is water depth; $\mathrm{g}$ is gravity acceleration; $\mathrm{C}$ is Chezy roughness coefficient; $\varepsilon$ is depth eddy viscosity; $\mathrm{f}$ is Coriolis parameter; $\mathrm{U}$ and $\mathrm{V}$ are depth-averaged velocity components in the $\xi$-direction and $\eta$ direction respectively; $\varphi$ is the pollutant concentration; $D_{\xi}, D_{\eta}$ are mixing coefficients in the $\xi$ and $\eta$ direction, respectively; $S_{\phi}$ is the source term. The velocities u (in the xdirection) and $\mathrm{v}$ (in the $\mathrm{y}$-direction) are related to $\mathrm{U}$ and $\mathrm{V}$ by

$$
\begin{aligned}
& U=\left(y_{\eta} / g_{\xi}\right) u-\left(x_{\eta} / g_{\xi}\right) v \\
& V=-\left(y_{\eta} / g_{\xi}\right) u-\left(x_{\eta} / g_{\xi}\right) v
\end{aligned}
$$

\section{Numerical scheme}

The transformed governing equations (3a) (3c) are discretized on a staggered $(\xi, \eta)$ grid and solved using alternating direction implicit finite difference scheme. The velocity variables are fully staggered, and the water level modes are located at the center of the continuity flow cell as illustrated in Fig. 2.

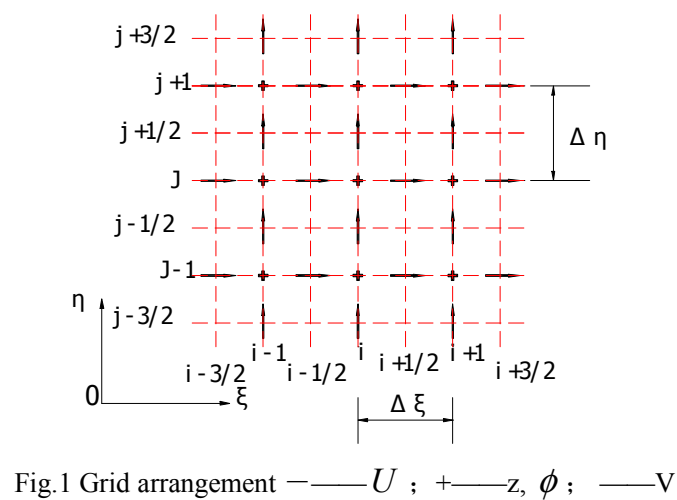

$\Delta \xi$ and $\Delta \eta$ are defined as the distances in the transformed domain between the velocity vector positions. Since the range of the coordinates $\xi$ and $\eta$ ind the computational plane is completely arbitrary, the mesh increments $\Delta \xi$ and $\Delta \eta$ are specified, for convenience, as unity.

A further index, $n$, is now introduced to denote the time level of the discretized hydrodynamic variables. The alternating direction algorithm splits each time step into two intervals. The procedure can be demonstrated with respect to the transformed equations as follows.

Step 1, in the first half time step:

Continuity equation ( $3 \mathrm{a}$ ) becomes

$$
\frac{\partial z^{n+\frac{1}{2}}}{\partial t}+\frac{1}{g_{\xi} g_{\eta}} \frac{\partial}{\partial \xi}\left(H^{n} U^{n+1} g_{\eta}\right)+\frac{1}{g_{\xi} g_{\eta}} \frac{\partial}{\partial \eta}\left(H^{n} V^{n} g_{\eta}\right)=0
$$

$\xi$-momentum equation ( $3 b)$ becomes

$$
\begin{aligned}
& \frac{\partial U^{n+1}}{\partial t}+\frac{U^{n+1}}{g_{\xi}} \frac{\partial U^{n}}{\partial \xi}+\frac{V^{n}}{g_{\eta}} \frac{\partial U^{n}}{\partial \eta}+\frac{\partial U^{n+1} V^{n}}{g_{\xi} g_{\eta}} \frac{\partial g_{\xi}}{\partial \eta}- \\
& \frac{\left(V^{n}\right)^{2}}{\partial \eta} \frac{\partial g_{\eta}}{\partial \xi}+g \frac{U^{n} \sqrt{\left(U^{n}\right)^{2}+\left(V^{n}\right)^{2}}}{C^{2} H}-f V^{n}+\frac{g}{g_{\xi}} \frac{\partial z^{n+\frac{1}{2}}}{\partial \xi} \\
& =\varepsilon\left(\frac{1}{g_{\xi}} \frac{\partial A^{n}}{\partial \xi}-\frac{1}{g_{\eta}} \frac{\partial B^{n}}{\partial \eta}\right)
\end{aligned}
$$

Step2, in the second half-time step:

Continuity equation ( $3 \mathrm{a}$ ) becomes

$$
\frac{\partial z^{n+1}}{\partial t}+\frac{1}{g_{\xi} g_{\eta}} \frac{\partial}{\partial \xi}\left(H^{n+\frac{1}{2}} U^{n+1} g_{\eta}\right)+\frac{1}{g_{\xi} g_{\eta}} \frac{\partial}{\partial \eta}\left(H^{n+\frac{1}{2}} V^{n+1} g_{\xi}\right)=0
$$


$\eta$-momentum equation (3c) becomes

$$
\begin{aligned}
& \frac{\partial V^{n+1}}{\partial t}+\frac{U^{n+1}}{g_{\xi}} \frac{\partial V^{n}}{\partial \xi}+\frac{V^{n+1}}{g_{\eta}} \frac{\partial V^{n}}{\partial \eta}+\frac{U^{n+1} V^{n+1}}{g_{\xi} g_{\eta}} \frac{\partial g_{\eta}}{\partial \xi} \\
& -\frac{\left(U^{n+1}\right)^{2}}{g_{\xi} g_{\eta}}+g \frac{V^{n} \sqrt{\left(U^{n+1}\right)^{2}+\left(V^{n}\right)^{2}}}{C^{2} H^{n+\frac{1}{2}}}+f U^{n+1}+\frac{g}{g_{\eta}} \frac{\partial z^{n+1}}{\partial \eta} \\
& =\varepsilon\left(\frac{1}{g_{\eta}} \frac{\partial A^{n}}{\partial \eta}+\frac{1}{g_{\xi}} \frac{\partial B^{n}}{\partial \xi}\right)
\end{aligned}
$$

By adding the above equations for the two half-time steps, it can be shown that the combined effect steps 1 and 2 results are the final results $\left(U^{n+1}, z^{n+1} a n d V^{n+1}\right)$ in one time step (from $\mathrm{t}=\mathrm{n} \Delta \mathrm{t}$ to $\mathrm{t}=(\mathrm{n}+1) \Delta \mathrm{t})$

\section{Numerical discretization}

When we solve the equations $(3 a) \sim(3 c)$, the viscosity of water is neglected. For brevity, only the $\xi$-direction ADI discretization of the continuity equation and $\xi$-momentum equation will be presented. A more detailed discussion of computational scheme can be found in Ref. [2].

In discretizing the equations (5), the average and finite difference of the function $F$ (such as $U, V$ and $z$, etc.) may be represented as

$$
\begin{gathered}
\bar{F}_{i, j}^{\xi}=\frac{1}{2}\left(F_{i-\frac{1}{2}, j}+F_{i+\frac{1}{2}, j}\right) \\
\bar{F}_{i, j}^{\eta}=\frac{1}{2}\left(F_{i, j-\frac{1}{2}}+F_{i, j+\frac{1}{2}}\right) \\
\bar{F}_{i, j}=\frac{1}{4}\left(F_{i-\frac{1}{2}, j-\frac{1}{2}}+F_{i-\frac{1}{2}, j+\frac{1}{2}}+F_{i+\frac{1}{2}, j-\frac{1}{2}}+F_{i+\frac{1}{2}, j+\frac{1}{2}}\right) \\
\frac{\partial F_{i, j}}{\partial \xi}=\frac{1}{\Delta \xi}\left(F_{i+\frac{1}{2}, j}-F_{i-\frac{1}{2}, j}\right) \\
\frac{\partial F_{i, j}}{\partial \xi_{i, j}}=\frac{1}{\Delta \eta}\left(F_{i, j+\frac{1}{2}}-F_{i, j-\frac{1}{2}}\right)
\end{gathered}
$$

Thus the transformed continuity equation for the first halftime step may be discretized and rearranged to give

$$
A_{1} U_{i-1 / 2, j}^{n+1}+B_{1} z_{i, j}^{n+1 / 2}+C_{1} U_{i+1 / 2, j}^{n+1}=D_{1}
$$

where

$$
\begin{aligned}
& A_{1}=-\frac{1}{J_{i, j}} \bar{H}_{i-1 / 2, j}^{\xi^{n}} g_{\eta i-1 / 2, j} ; B_{1}=\frac{2.0}{\Delta t} ; C_{1}=\frac{1}{J_{i, j}} \bar{H}_{i+1 / 2, j}^{\xi^{n}} g_{\eta i+1 / 2, j} ; \\
& D_{1}=\frac{2 z_{i, j}^{n}}{\Delta t}-\frac{1}{J_{i, j}}\left[\bar{H}_{i+1 / 2, j}^{\xi^{n}} V_{i, j+1 / 2}^{n} g_{\eta i, j+1 / 2, j}-\bar{H}_{i, j-1 / 2, j}^{\eta^{n}} V_{i, j-1 / 2}^{n} g_{\eta i, j-1 / 2}\right]
\end{aligned}
$$

And the transformed $\xi$-momentum equation for the first half-time step may be discretized and rearranged to give

$$
A_{2} z_{i, j}^{n+1 / 2}+B_{2} U_{i+1 / 2, j}^{n+1}+C_{2} z_{i+1, j}^{n+1 / 2}=D_{2}
$$

where

$$
\begin{aligned}
& A_{2}=-\frac{g}{g_{\xi i+1 / 2, j}} ; B_{2}=\frac{2.0}{\Delta t}+\frac{1}{g_{\xi i+1 / 2, j}}<\frac{\partial U^{n}}{\partial \xi}>+ \\
& \frac{\bar{V}_{i+1 / 2, j}^{n}\left(g_{\xi i+1 / 2, j+1 / 2}-g_{\xi+1 / 2, j-1 / 2}\right)}{J_{i+1 / 2, j}} ; \\
& C_{2}=\frac{g}{g_{\xi i+1 / 2, j}} ; D_{2}=\frac{U_{i+1 / 2, j}^{n}}{\Delta t}-\frac{\bar{V}_{i+1 / 2, j}^{n}}{g_{\eta i+1 / 2, j}}<\frac{\partial U^{n}}{\partial \eta}>+ \\
& \frac{\left(\bar{V}_{i+1 / 2, j}^{n}\right)^{2}}{J_{i+1 / 2, j}}\left(g_{\eta i+1, j}-g_{\eta i, j}\right) \\
& -g \frac{U_{i+1 / 2, j}^{n}\left[\left(U_{i+1 / 2, j}^{n}\right)^{2}+\left(\bar{V}_{i+1 / 2, j}^{n}\right)^{2}\right]^{1 / 2}}{C^{2} \bar{H}_{i+1 / 2, j}^{\pi}}+f \bar{V}_{i+1 / 2, j}^{n}+ \\
& \frac{\varepsilon}{g_{\xi}}\left[A_{i+1 / 2, j}^{n}-A_{i-1 / 2, j}^{n}\right]+\frac{\varepsilon}{g_{\eta}}\left[B_{i, j+1 / 2}^{n}-B_{i, j-1 / 2}^{n}\right]
\end{aligned}
$$

During the above $\xi$-direction release the $\xi$-momentum cross-convective terms $<>$ are differenced using a two-order upwind scheme and discretized as

$$
<\frac{\partial U^{n}}{\partial \eta}>=\left\{\begin{array}{lll}
\frac{3}{2} U_{i, j}^{n}-2 U_{i, j-\frac{1}{2}}^{n}+\frac{1}{2} U_{i, j-1}^{n} & \text { if } & \bar{V}_{i+\frac{1}{2}, j}^{n} \geq 0 \\
-\frac{3}{2} U_{i, j}^{n}+2 U_{i, j+\frac{1}{2}}^{n}+\frac{1}{2} U_{i, j+1}^{n} & \text { if } & \bar{V}_{i+\frac{1}{2}, j}^{n}<0
\end{array}\right.
$$

and $<\frac{\partial U^{n}}{\partial \xi}>=\left\{\begin{array}{lr}\frac{3}{2} U_{i, j}^{n}-2 U_{i-\frac{1}{2}, j}^{n}+\frac{1}{2} U_{i-1, j}^{n} & \text { if } \quad U_{i-\frac{1}{2}, j}^{n} \geq 0 \\ -\frac{3}{2} U_{i, j}^{n}+2 U_{i-\frac{1}{2}, j}^{n}+\frac{1}{2} U_{i-1, j}^{n} & \text { if }\end{array} \quad U_{i-\frac{1}{2}, j}^{n}<0\right.$

Using the ADI method to solve the tri-diagonal matrix equations about $\mathrm{U}$ and $\mathrm{z}$, we can obtain the values of $\mathrm{U}^{\mathrm{n}+1}$ and $\mathrm{z}^{\mathrm{n}+0.5}$. These are the results for the first half-time step; and the results are prepared for the initial values of the second step. And then, using the similar method, we can obtain the values of $\mathrm{V}^{\mathrm{n}+1}$ and $\mathrm{z}^{\mathrm{n}+1}$. Up to now, final results $\left(\mathrm{U}^{\mathrm{n}+1} 、 \mathrm{~V}^{\mathrm{n}+1}\right.$ and $\left.\mathrm{z}^{\mathrm{n}+1}\right)$ are obtained for one time step. When the values of $U, V$ and $Z$ 
are obtained, we use upwind difference scheme to discretize the convective term and central difference scheme to discretize the diffusion term in equation ( $3 \mathrm{~d})$, and can obtain the value of $\varphi$ at each time step.

\section{E. Boundary Conditions}

The boundary conditions for flow are that the upstream and downstream water level is specified and the flux through the solid boundaries is zero; and the initial conditions are that water level at any flow element is the averaged value of upstream and downstream water level and velocity is set equal to zero.

The boundary conditions for water quality are that the pollutant concentration $\varphi$ through the solid boundaries is zero, and at the inlet boundary and at the outlet boundary it doesn't change in the flowing direction and at the discharge place is specified. The initial condition is that $\varphi$ is set to be zero.

\section{RESULTS OF COMPUTATION}

This method has been used to predict the water quality for the HanJiang XianTao river flow. The water quality measurement station for XianTao river flow is located on the bend river in the vicinity of XianTao fertilizer plant, and the measurement items for the water quality are $\mathrm{PH}, \mathrm{COD} c r, \mathrm{CODmn}, \mathrm{BODs}, \mathrm{NH}_{3}$, and $\mathrm{P}$, etc. The polluted water discharge is about $1170 \times 10^{8} \mathrm{Kg}$ each year, and is one of the greatest pollution sources in the main stream for HanJing flow. Here we compute the distribution of the concentration of COD and compare with the measurement data. A fair agreement between the values measured and computed demonstrates the validity of the method development.

The computed region for HanJiang XianTao river flow with bend river boundaries is about $3.01 \mathrm{Km}$ long and $250 \mathrm{~m}$ wide, shown in Fig. 2. The region concerned is divided into 4200 elements. In the computation, the time step $\Delta t$ is about $15 \mathrm{~s}$; the size of space step $\Delta s$ is about from 20 meters to 150 meters; the Courant number is less than 5 ; the Coriolis parameter $\mathrm{f}$ is about $7.37 \times 10^{-5}$; the roughness $\mathrm{n}$ is about 0.014 .

The measurement values of velocity and water depth on cross section $\mathrm{k}_{1}$ are specified for the upstream boundary conditions. The important hydraulic parameters such as velocity and water level and pollutant concentration have been obtained. The computed results are shown in Fig.3-Fig.4. The computed results and measured data show that the polluted water area is about 800 meters long and 70 meter wide. The comparison between the computed and measured data shows that the errors on $\mathrm{k}_{3}$ cross-section is larger and on others are smaller. Fig. 2 shows computed region; Fig. 3 shows comparisons of velocity on cross section $k_{2}$ between computed results and measured data. Fig.4 shows comparisons of the distribution of computational pollutant concentration of COD on cross section $\mathrm{k}_{2}$.

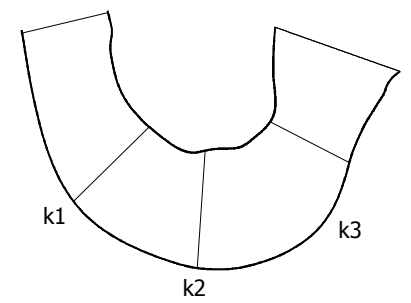

Fig. 2 shows computed region

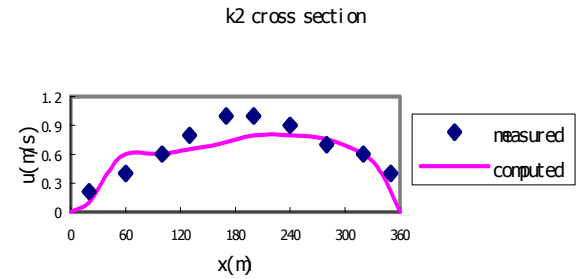

Fig. 3 Comparisons of velocity on cross section $k_{2}$ between computed and measured data

k2 cross-secti on

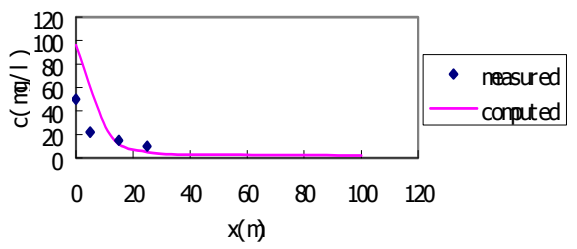

Fig. 4 Comparisons of pollutant concentration of COD on cross-section $\mathrm{k}_{2}$ between computed and measured data

\section{CONCLUSIONS}

Some advanced techniques of computational fluids dynamics, such as the technique of orthogonal curvilinear grid generation and the technique of moving boundary, presented above are convenient and effective in dealing with the complicated boundary of physical region and the computational finite cell exposed to water surface. The method of alternating direction implicit finite difference scheme used to solve the partial differential equations has good stability, convergence, and accuracy. This mathematical model can be used to accurately predict the water quality for tidal rivers.

\section{Acknowledgments}

This work was supported by the Chinese post-doctorate science Foundation (20090451163), the Jiangsu Province postdoctorate Foundation (0901006C), and the Shanxi Province key subject construction funds.

\section{REFERENCES}

[1] J.F.Thompson, "Numerical Solution of Flow Problems Using BodyFitted Coordinate Systems", Computational Fluid Dynamics, 1980, pp. $1-98$

[2] Wei Wenli, Liu Yuling, Li Jianzhong, "The Application of Orthogonal Boundary-Fitted Coordinate System and Fractional Step Method to the Calculation of 2D Flow Field in River Course", Journal of Xi'an University of Technology, 1996 (in Chinese)

[3] Wei wenli, "Numerical Solution for Unsteady 2-D Flow Using the Transformed Shallow Water Equations", Journal of Hydrodynamics, Ser. B,1993(3), pp.65-71.

[4] Wei WenLi, "Study on Curvilinear Grid Generation", 9th national Hydrodynamics Conference, Nanjing, China, 1995, pp.162 166 (in Chinese) 